\title{
O trabalho multiprofissional nos cuidados paliativos: um diálogo entre a medicina funcional integrativa $e$ a atençãopsicológica
}

\author{
Multiprofessional work in palliative care: a dialogue between integrative functional \\ medicine and psychological care
}
Trabajo multiprofesional en cuidados paliativos: un diálogo entre la medicina funcional integradora y la atención psicológica

Caroline Wolff ${ }^{1 *}$, Dafne Andreatta Braganholo ${ }^{1}$,Gabriela Vasco da Silva ${ }^{1}$, Jeanne Christini da Silva Rosa $^{2}$, Laura Terezinha Velho Fidelis ${ }^{1}$, Luiza Leal de Faria Garcia ${ }^{1}$, Lygia Eduarda de Menezes Moraes Teixeira ${ }^{2}$, Maria Luisa Graciosa Birro ${ }^{1}$, Sol Berenice Tambone ${ }^{1}$, Márcia Farsura de Oliveira ${ }^{3}$.

\section{RESUMO}

Objetivo: Identificar a importância da prática dos cuidados paliativos nos tratamentos de pacientes terminais, visando compreender e satisfazer todas as necessidades das esferas psicoemocional, orgânica, social e espiritual. Revisão bibliográfica: Observou-se que os cuidados paliativos são de vital importância, uma vez que se preocupam em promover a qualidade de vida dos pacientes e familiares, com sensibilidade de considerar diversos aspectos da vida dessas pessoas, preparados no enfrentamento das adversidades que implicam um tratamento terminal. É importante salientar também que a presença multiprofissional se diz fundamental para maior compreensão dos casos e manejo clínico adequado, mesmo que ocorram dentro de ambientes hospitalares. Considerações Finais: Finalmente, salienta-se o reconhecimento da união entre Psicologia e Medicina, por exemplo, demonstra que o bem-estar do paciente não pode ser limitado em apenas um departamento da saúde, por isso que a comunidade científica deve estar atenta aos efeitos benéficos da prática dos cuidados paliativos como uma forma de tratamento de escuta ativa, atenção integral, e preocupação ao direito básico à dignidade humana.

Palavras-chave: Cuidados paliativos, Equipe de assistência ao paciente, Cuidados paliativos na terminalidade da vida.

\section{ABSTRACT}

Objective: To identify the importance of the practice of palliative care in the treatment of terminal patients, aiming to understand and meet all the needs of the psycho-emotional, organic, social and spiritual spheres. Bibliographic review: It was observed that palliative care is of vital importance, since it is concerned with promoting the quality of life of patients and family members, with sensitivity to consider various aspects of the lives of these people, prepared in facing the adversities that involve terminal treatment. It is also important to point out that the multiprofessional presence is fundamental for a better understanding of the cases and an adequate clinical management, even if they occurinside hospital environments. Final considerations: Finally, the recognition of the union between Psychology and Medicine, for example, demonstrates that the well being of the patient cannot be limited in only one department of health, so the scientific community must be aware of the beneficial effects of the practice of palliative care as a form of treatment of active listening, integral attention, and concern for the basic right to human dignity.

Key words: Palliative care, Patient care team, Hospice care.

${ }^{1}$ Faculdade de Ciências Sociais de Santa Catarina (CESUSC), Florianópolis - SC.

*E-mail: carolinewolff@outlook.com

2 Universidade do Sul de Santa Catarina - Pedra Branca (UNISUL-PB), Palhoça - SC.

${ }^{3}$ Faculdade Dinâmica do Vale do Piranga (FADIP), Ponte Nova - MG. 


\section{RESUMEN}

Objetivo: Identificar la importancia de la práctica de los cuidados paliativos en los tratamientos de pacientes terminales, con el fin de comprender y satisfacer todas las necesidades de las esferas psicoemocional, orgánica, social y espiritual. Revisión bibliográfica: Se observó que los cuidados paliativos son de vital importancia, ya que se ocupan de promover la calidad de vida de los pacientes y familiares, con sensibilidad para considerar diversos aspectos de la vida de estas personas, preparadas para enfrentar las adversidades que implica un tratamiento terminal. También es importante destacar que la presencia multiprofesional es fundamental para una mejor comprensión de los casos y un manejo clínico adecuado, aunque se produzcan en el ámbito hospitalario. Consideraciones finales: Por último, se destaca el reconocimiento de la unión entre la Psicología y la Medicina, por ejemplo, demuestra que el bienestar del paciente no puede limitarse en un solo departamento de la salud, por lo que la comunidad científica debe estar atenta a los efectos benéficos de la práctica de los cuidados paliativos como una forma de tratamiento de escucha activa, atención integral y preocupación por el derecho básico a la dignidad humana.

Palabras clave: Cuidados paliativos, Grupo de atención al paciente, Cuidados paliativos al final de la vida.

\section{INTRODUÇÃO}

Ao se falar em "cuidados paliativos" remete-se a todo cuidado, amparo e abrigo que essa filosofia diz respeito quando a cura de determinada doença não é mais possível. A terminologia vem do termo hospice, antigos abrigos destinados ao conforto e a cuidados com peregrinos e doentes que muitas vezes morriam nesses locais, e de pallium, que em latim significa manto (FRANKLING H, et al., 2021).

O Movimento Hospice foi iniciado pela enfermeira, assistente social e médica inglesa Cicely Saunders que, em meados do século $X X$, ao trabalhar com pacientes em estado terminal e presenciando o sof rimento humano, passou a se dedicar à sistematização de fundamentos voltados para o alívio da dor e do sof rimento inerentes ao final da vida. Desta forma o objetivo visa sempre a compreensão total e integral das necessidades do paciente, do qual se concilia os aspectos orgânico, psicoemocional, social e espiritual da pessoa doente e dos que participam de sua vida. E assim, em 1967, em Londres, fundou St. Christhopher Hospice (NICKEL L, et al., 2016).

A primeira definição de cuidados paliativos foi publicada em 1986 pela OMS (MINISTÉRIO DA SAÚDE, 1986). De acordo com essa definição, os cuidados paliativos (CP) eram destinados a pacientes fora da possibilidade de cura, cuidados esses, ativo e total, para controle da dor e outros sintomas, assim como problemas psicossociais e espirituais, objetivando a melhora da qualidade de vida para o $p$ aciente e para todo seu entorno, inclusive para sua família. No inicial tal definição era apenas voltada para tratamento com pacientes com câncer, preconizando-os na assistência integral visando os cuidados de final de vida (FRANKLING $\mathrm{H}$, et al., 2021).

Assim, em 2002, a definição de CP foi revisada pela Organização Mundial da Saúde, ampliando o conceito, de forma a contemplar todas as doenças e aplicável o mais precocemente possível, incluindo o período de luto (WORLD HEALTH ORGANIZATION, 2002). Atualmente se compreende a partir da OMS de uma forma mais integrativa, ampliando-se o que se pensa ao falar e trabalhar em "cuidados paliativos", diante disso 0 mesmo pode ser sempre adaptado às singularidades relacionadas ao local, aos recursos disponíveis e ao perfil epidemiológico dos grupos atendidos. A demanda relacionada a esses pacientes e o número de unidades especializadas vem crescendo, assim como as discussões sobre o tema (NICKEL L, et al., 2016).

Atualmente, poucos países adotam uma política pública de saúde que abrange os cuidados paliativos, com destaque para o Brasil, que of erece esses serviços como parte da atenção continuada integrada no âmbito do Sistema Único de Saúde (SUS). Os cuidados paliativos costumam ser prestados por meio dos Serviços de Atenção Domiciliar (SAD), que atendem os pacientes em suas próprias residências, junto à família e aos amigos, não sendo obrigados a se adaptar à rotina e ao ambiente hospitalar. Nesse caso, não é possível 
esclarecer a importância da prestação desses serviços para melhorar a qualidade de vida dos pacientes em fase terminal (CARLO MMRP e LIMA NKC, 2015).

Sendo assim, a rede de cuidados paliativos deve estruturar-se de forma a articular os serviços e estratégias de: (i) atenção básica (alternativa assistencial fora do ambiente hospitalar), (ii) atenção domiciliar of erecida pela atenção básica ou hospitais (atenção no domicílio, classificada como visita, atendimento ou internação); (iii) instituições de longa permanência (ILP) ou unidades de retaguarda (que disponibilizam internações por período superior a 45 dias); e (iv) atenção hospitalar (assistência multiprofissional realizado em diferentes tipos de unidades hospitalares) (ALI AMASA, et al., 2012).

Diante de todas essas definições, os cuidados paliativos consistem na assistência prestada por uma equipe multiprofissional e interdisciplinar de cuidados totais e integrais, com o objetivo de promover e assegurar a qualidade de vida das pessoas assistidas e de seus familiares que passam pela situação de doença potencialmente fatal, desde o diagnóstico até o período de suporte ao luto, por meio de avaliações corretas e de tratamentos adequados, fortalecendo o vínculo e adquirindo confiança para continuar com a assistência da melhor forma. Quando há poucas possibilidades de resposta à terapêutica curativa, evolução clínica oscilante, impacto emocional e social para o doente e família e prognóstico de vida limitado, há necessidade de adequação terapêutica, que é vista dentro da perspectiva dos cuidados paliativos (MACIEL MGS, et al., 2006).

É importante destacar que, as pessoas nesse estágio precisam de um apoio que vai além da intervenção biomédica: uma atenção integrada e organizada, com modelos de atenção à saúde (MENDES EV, 2010). Sendo assim, os profissionais da saúde devem entendê-los como núcleo gerador das decisões e terem conforme perspectiva central a ajuda para que esse indivíduo enfermo possa viver ativa e intensamente até sua óbito, com dignidade e respeito. Além disso, a equipe composta deve defender o direito à vida em sua plenitude, destacando os direitos à informação sobre o percurso da doença, à autonomia para decisões quanto ao tratamento, o alívio do sofrimento e dos sintomas angustiantes de difícil controle, integrando aspectos psicossociais e espirituais na assistência, andando em conjunto com outras terapias, como por exemplo, quimioterapia e radioterapia (CARLO MMRP e LIMA NKC, 2015).

Diante do exposto, o objetivo do presente trabalho foi identificar a importância da prática dos cuidados paliativos nos tratamentos de pacientes terminais, considerada uma abordagem, composta por uma equipe multidisciplinar, que se preocupa em ter um olhar humano quanto ao sofrimento dos envolvidos, visando compreender e satisfazer todas as necessidades das esferas psicoemocional, orgânica, social e espiritual.

\section{REVISÃO BIBLIOGRÁFICA}

\section{Os Cuidados Paliativos no Brasil}

Durante a década de 1980 e com o fim do regime militar, os trabalhos em Cuidados Paliativos (CP) passaram a circular no território brasileiro. Após um momento trágico em que milhares de pessoas morrem sem ao menos conhecerem seus quadros clínicos, passaram a surgir os primeiros serviços em CP do país, tendo como marco a criação em 1991 do primeiro serviço oficial no Instituto Nacional de Câncer (INCA) (PAIVA CF, et al., 2021).

Em 1997, com a crescente deste movimento, os profissionais da área criaram a Associações Brasileiras de Cuidados Paliativos (ABCP) para ampliação e regulamentação desta área, porém só em 2002 o serviço passou a ser oferecido também pelo Sistema Único de Saúde (SUS) (NICKEL L, et al., 2016).

De acordo com a Organização Mundial da Saúde (OMS) os Cuidados Paliativos dizem respeito "às ações ativas e integrais prestadas a pacientes com doença progressiva e irreversível e a seus familiares" sendo essencial a atenção sobre a dor e sintomas como forma de controle, prevenção e alívio, além de buscar uma maior compreensão sobre eles (PEREIRA CA e RIBEIRO JFS, 2019). O CP não se resume exclusivamente a atenção relacionada a doenças crônicas, degenerativas e do envelhecimeno, porém é dentro desse setor que eles alcançam maiores aplicabilidades (NICKEL L, et al., 2016). 
A presença multiprofissional se diz fundamental para maior compreensão dos casos e manejo clínico adequado, mesmo que ocorram dentro de ambientes hospitalares. Desta forma, o foco nos CP deve circular em torno da promoção de qualidade de vida e humanização do olhar. Isso evidencia a necessidade de um diálogo entre medicina e psicologia, afinal, os sintomas enf rentados pelos sujeitos dizem respeito a graves enfermidades, que implicam diretamente no funcionamento físico, social e emocional (PAIVA CF, et al, 2021).

\section{Atenção Psicológica nos Cuidados Paliativos}

O paciente que está por receber o diagnóstico de uma doença incurável e que está fora de possibilidade terapêutica é chamado de "paciente terminal". Esta denominação nos remete a ideia de que não há mais nada que se possa fazer por este paciente. Entretanto, é necessário salientar que, mesmo na fase final da vida, este paciente ainda vive, possui desejos e necessidades que podem Ihe propiciar bem -estar e conforto. A fim de que isso ocorra, é necessário que a equipe multiprofissional esteja presente para atuar de forma integral. Portanto, dentro da equipe multiprofissional, o psicólogo tem uma das principais funções perante o paciente, a qual refere-se a escutar e acolher a dor, com o intuito de auxiliá-lo a ressignificar a vida e a morte durante este período (ESSLINGER I, 2004). Assim, na atuação do psicólogo é necessário contemplar as questões envolvidas na queixa do paciente, assim como em seus sintomas e patologia, possibilitando, desta maneira, uma atenção total neste processo (OTHERO MB e COSTA DG, 2007).

À vista disso, o psicólogo tem o papel significativo de abrir caminho para uma comunicação entre o paciente, seus familiares e a equipe, para que seja possível identificar o que o paciente e a família mais precisam naquele momento, com o objetivo de ampliar seu bem-estar; detectar medos e inseguranças, buscando desta forma promover apoio de acordo com os valores espirituais e culturais daquele paciente; mediar oportunidades para que temas pendentes sejam discutidos, assim como contribuir na relação entre equipe multiprofissional, paciente e familiares (OTHERO MB e COSTA DG, 2007).

Ademais, o psicólogo, por meio da escuta ativa e comunicação eficiente, consegue compreender o paciente como um ser em sua totalidade, um sujeito que sofre, mas que para além da sua dorfísica, existe este paciente com diversas questões existenciais e necessidades que, muitas vezes, fármacos e/ou equipamentos tecnológicos não conseguem atender (PEREIRA CA e RIBEIRO JFS, 2019). Além disso, a atuação do psicólogo em CP é mostrar ao paciente que é possível partilhar o momento vivido, buscando e conhecendo seus recursos internos para que dessa forma possa lidar com seus sentimentos, trabalhando com ele o sofrimento psíquico, o qual inclui uma série de sentimentos e emoções, como ansiedade, depressão, dignidade af etada e seus medos - possibilitando assim um significado dif erente para a experiência de adoecer (PEREIRA CA e RIBEIRO JFS, 2019).

Vale ressaltar que de acordo com a Academia Nacional de Cuidados Paliativos (2012) o psicólogo além de dar assistência aos pacientes e às famílias, também dá atenção à equipe multiprofissional com a finalidade de acolher todas as demandas deste e promover um espaço apropriado, bem como apresentar todas as informações necessárias à equipe a respeito do paciente possibilitando assim uma interação ideal entre paciente, familiares e equipe (DANTAS MMF e AMAZONAS MCLA, 2016). Por fim, nota-se a importância que a atenção psicológica possui nos Cuidados Paliativos em todas as diferentes etapas que o paciente e os familiares percorrem durante este período, com o propósito de manter o bem-estar psicológico de todos os envolvidos, bem como identificar as questões emocionais que possam prejudicar o processo de tratamento (BEN-ARYE E, et al., 2018).

\section{Medicina Complementar e Integrativa nos Cuidados Paliativos}

A Medicina Complementar e Integrativa (CIM) está se tornando mais presente entre os pacientes oncológicos e cada vez mais centros de oncologia fornecem CIM como parte de seu serviço paliativo. Nos pacientes paliativos, a fadiga é uma das maiores queixas relacionada ao câncer, estando associada a fatores relacionados ao tumor, bem como a toxicidades relacionadas à quimioterapia (anemia, sintomas gastrointestinais com perda de peso etc.), preocupações emocionais e dificuldades relacionadas ao sono. Diante disso, foram consideradas benéficas terapias como a acupuntura, acupressão, moxabustão a laser infravermelho, Qigong, e Tai Chi (BEN-ARYE E, et al., 2018). 
Há vários estudos sobre cuidados paliativos abordando a oncologia integrativa, que oferecem um amplo espectro de terapias da medicina complementar, muitas das quais podem aumentar os ef eitos dos cuidados de suporte e paliativos para pacientes com câncer ginecológico. Dessa forma, a oncologia utiliza dos princípios da medicina integrativa, com foco em aliviar o sofrimento dos pacientes, reduzindo os ef eitos adversos da quimioterapia e da radiação, melhorando a qualidade de vida e a autonomia.

Muitos pacientes com câncer ginecológico consideram o aconselhamento nutricional como parte da consulta oncológica integrativa. A medicina funcional reconhece a importância da dieta no risco de doença primária e recorrente, bem como nos resultados relacionados à qualidade de vida $(\mathrm{QV})$ durante o tratamento (BEN-ARYE E, et al., 2018). Com base nisso, a Curcumina é um exemplo de fitoquímico capaz de inibir os ef eitos de agentes quimioterápicos, como vimblastina, em células de câncer cervical (DANG YP, et al. 2015). Já o uso de probióticos vivos, como Lactobacillus acidophilus e Bifidobacterium bifidum, demonstrou reduzir a incidência de diarréia induzida por radiação e a necessidade de medicação antidiarreica em pacientes em tratamento com cisplatina e radioterapia pélvica para câncer cervical localmente avançado (LINN YH, et al., 2019).

Existem ainda outros estudos clínicos, um deles demonstrou que o visco (Viscum album) pode aumentar o tempo de sobrevida em pacientes com câncer de pulmão de células não pequenas, como adenocarcinoma (SCHAD F, et al., 2018). Enquanto outro ensaio clínico randomizado e controlado da Coreia mostrou que o Ginseng vermelho (Panax ginseng Meyer) reduziu os sintomas de fadiga, náusea e dispneia em pacientes submetidas à quimioterapia adjuvante para câncer epitelial de ovário (KIM HS, et al., 2017).

Além de um estudo cruzado da Tailândia mostrando que a acupuntura foi tão eficaz quanto o ondansetrona na prevenção de vômitos de início imediato (dentro de 24 h) após um regime de carboplatinapaclitaxel para câncer de ovário, e superior à droga na prevenção de vômitos tardios. O grupo tratado com acupuntura também relatou significativamente menos efeitos adversos, como insônia e constipação, e melhores pontuações físicas, sociais e gerais de bem-estar, além de menor custo de tratamento comparado ao grupo de controle (LIU YQ, et al., 2015).

Ressalta-se a atividade física como um fator importante para a QV durante a quimioterapia para pacientes com câncer de ovário, um ensaio clínico randomizado na China demonstrou que um enfermeiro domiciliar, o programa de exercícios e terapia cognitivo-comportamental para pacientes com câncer de ovário submetidas à quimioterapia resultou em uma redução da fadiga e depressão relacionadas ao câncer e melhora na qualidade do sono (CRAWFORD JJ, et al., 2015).

Enquanto outro estudo analisou pacientes com tumores pulmonares e/ou gastrointestinais avançados localmente ou metastáticos que estavam sob cuidados paliativos, como acompanhamento nutricional e sessões de exercício físico, observando uma melhora da qualidade de vida geral, uma vez que aumentou a ingestão de proteínas desses pacientes e reduziu sintomas como náuseas e vômitos (USTER A, et al., 2018).

\section{Vitaminas Hidrossolúveis}

No que diz respeito às vitaminas hidrossolúveis, como as do complexo $B$ e vitamina $C$, alguns estudos apontam a deficiência delas como algo muito frequente dentre os pacientes sob cuidados paliativos. Um estudo publicado por Vollbracht C, et al. (2019) apontou que 68\% dos pacientes internados no Hospital Geral da Alemanha sob cuidados paliativos tinham deficiência de mais de 1 vitamina; este mesmo estudo trouxe os seguintes resultados: $48 \%$ tinham deficiência de vitamina B6, $45 \%$ tinham deficiência de vitamina $C, 26 \%$ com deficiência de vitamina B1 e 13\% com deficiência de vitamina B12. Com isso, a partir dos resultados foi possível apontar uma relação dos baixos níveis de B1 com o aumento da depressão e da percepção dolorosa (VOLLBRACHT C, et al., 2019).

\section{Vitamina D}

A vitamina $D$ é um hormônio esteroide com múltiplas funções no corpo humano, dentre essas funções tem-se a ação anti-inflamatória, a qual em pacientes gravemente enfermos sob cuidados paliativos é de grande valia. Todos os estudos revisados no presente trabalho correlacionaram o sintoma de fadiga com 
baixos níveis de Vitamina $D$, e a partir da reposição os pacientes relataram melhora da disposição e de sintomas depressivos (MARTíNEZ-ALONSO M, et al., 2015).

É esperado que os pacientes oncológicos apresentem baixos níveis de Vitamina $\mathrm{D}$, uma vez que grande parte das vezes eles estão hospitalizados; além disso, é contraindicado a exposição à radiação solar antes de sessões de quimioterapia e radioterapia, o que corrobora para a deficiência dessa vit amina. A partir disso é necessário se avaliar alguns aspectos da vida do paciente oncológico sob cuidados paliativos, pois a Vitamina $D$ necessita não apenas da exposição solar, mas também de uma dieta adequada, com uma boa ingestão de proteínas e gorduras. No entanto, como muitos desses indivíduos têm dificuldades alimentares, seja pela incapacidade proporcionada pela própria doença, seja por ef eitos colaterais dos tratamentos, muitas vezes eles não possuem nem mesmo a matéria prima para produzir Vitamina $D$ bem como outros hormônios (VOLLBRACHT C, et al., 2019).

Já foi constatado que os ef eitos de toxicidade da vitamina $D$ são extremamente raros, como por exemplo a Hipercalcemia. Sendo assim, doses de $4.000 \mathrm{Ul}$ por dia se mostraram eficientes contra os sintomas de fadiga. Além disso, alguns estudos trouxeram dados da redução na dose de opióides de pacientes que iniciaram a suplementação com essa vitamina. Por todos esses pontos apresentados os estudos puderam concluir que a reposição de vitamina $D$ é benéfica para os pacientes em questão, tanto por melhorar os sintomas de fadiga como a qualidade de vida geral (FRANKLING $\mathrm{H}$, et al., 2021).

\section{CONSIDERAÇÕES FINAIS}

Por meio da análise bibliográfica desse estudo, compreende-se que os cuidados paliativos como promoção de qualidade de vida para o paciente e sua família, só é possível através de uma equipe multidisciplinarcapaz de contemplar o sujeito como um ser em sua totalidade considerando todos os diferentes aspectos que participam de sua vida. Além disso, observou-se a importância e a complementaridade na atuação da psicologia e medicina em relação aos $\mathrm{CP}$ e o propósito de manter o bem-estar psicológico de todos os envolvidos por meio do acolhimento e da escuta ativa. Confirmando, assim, a necessidade de um trabalho multidisciplinar para se conseguir alcançar uma assistência integral ao paciente e seus familiares. Reconhece se a necessidade do desenvolvimento de novas pesquisas, bem como maiores aprofundamentos e especificações da temática, visto que ainda é necessário discutir cada vez mais sobre os serviços paliativos, o qual poucos países praticam, visando difundir a ideia do direito a um tratamento voltado à atenç ão integrada e o respeito a vida.

\section{REFERÊNCIAS}

1. ALI AMASA, et al. Protocolo clínico e de regulação para atenção a pacientes em cuidados paliativos. In: SANTOS JS, et al. Protocolos clínicos e de regulação: acesso à rede de saúde. Rio de Janeiro: Els evier, 2012;141-160.

2. BEN-ARYE E, et al. The journal of alternative and complementary medicine, 2018;24(9-10):881-889.

3. BEN-ARYE E, et al. Inverse relationship between reduced fatigue and severity of anemia in oncology patients treated with integrative medicine: understanding the paradox. Support Care Cancer, 2018;26(12):4039-4048.

4. CARLO MMRP, LIMA NKC. Cuidados paliativos. In: LOPES AC. Tratado de clínica médica. Rio de Janeiro: Roca, 2015.

5. CRAWFORD JJ, et al. Associações entre exercício e crescimento pós-traumático em sobreviventes de câncer ginecológico. Support Care Cancer, 2015;23:705-714.

6. DANTAS MMF, AMAZONAS MCLA. A Experiência do Adoecer: Os Cuidados Paliativos diante da Impossibilidade da Cura. Revista da Escola de Enfermagem da USP, 2016;50(n.esp): 047-053.

7. DANG YP, et al. Curcum in improves the paclitaxel-induced apoptosis of HPV-positive human cervical cancer cells via the NF-kb-p53-caspase-3 pathway. Exp Ther Med, 2015;9(4): 1470-1476.

8. ESSLINGER I. De quem é a vida, afinal? Descortinando os Cenários da Morte no Hospital. São Paulo: Casa do Psicólogo, 2004.

9. FRANKLING H, et al. 'Palliative-D'-Vitamin D Supplementation to Palliative Cancer Patients: A Double Blind, Randomized Placebo-Controlled Multicenter Trial. Cancers, 2021;13: 3707.

10. KIM HS, et al. Effect of Red Ginseng on Genotoxicity and Health-Related Quality of Life after Adjuvant Chemotherapy in Patients with Epithelial Ovarian Cancer: A Randomized, Double Blind, Placebo-Controlled Trial. Nutrients, 2017; 9(7): 772. 
11. LINN YH, et al. Effect of Probiotics for the Prevention of Acute Radiation-Induced Diarrhoea Among Cervical Cancer Patients: a Randomized Double-Blind Placebo-Controlled Study. Probiotics Antimicrob Proteins, 2019; 11 (2): 638647.

12. LIU YQ, et al. Wrist-ankle acupuncture and ginger moxibustion for preventing gastrointestinal reactions to chemotherapy: A randomized controlled trial. Chin J Integr Med, 2015;21(9):697-702.

13. MACIEL MGS, et al. Critérios de qualidade para os cuidados paliativos no Brasil. Rio de Janeiro: Academia Nacional de Cuidados Paliativos, Diagraphic, 2006.

14. MARTÍNEZ-ALONSO M, et al. Vitamin D deficiency and its association with fatigue and quality of life in advanced cancer patients under palliative care: A cross-sectional study. Palliat Med., 2016;30(1):89-96.

15. MENDES EV. As redes de atenção à saúde. Ciência \& Saúde Coletiva, 2010;15(5):2297-2305.

16. MINISTÉRIO DA SAÚDE. Relatório Final da 8ª Conferência Nacional da Saúde. Brasília -DF: Brasil, 1986. Disponível em: https://bvsms.saude.gov.br/bvs/publicacoes/8_conferencia_nacional_saude_relatorio_final.pdf. Acessado em: 10 mar. 2021.

17. NICKEL L, et al. Grupos de pesquisa em cuidados paliativos: a realidade brasileira de 1994 a 2014 . Esc. Anna Nery, 2016;20(1):70-76.

18. PAIVA CF et al. Aspectos históricos no manejo da dor em cuidados paliativos em uma unidade de referência oncológica. Rev. Bras. Enferm, 2021; 74:05.

19. PEREIRA CA, RIBEIRO JFS. Cuidados paliativos: Reflexões sobre a Psicologia e os cuidados paliativos para pacientes e familiares, 2019;10(2).

20. OTHERO MB, Costa DG. Propostas desenvolvidas em cuidados paliativos em um hospital amparador - terapia ocupacional e psicologia. Revista prática Hospitalar, 2007;9(52):157-160.

21. SCHAD F, et al. Overall survival of stage IV non-small cell lung cancer patients treated with Viscum album L. In addition to chemotherapy, a real-world observational multicenter analysis, 2018;13(8).

22. USTER A, et al. Effects of nutrition and physical exercise intervention in palliative cancer patients: A randomized controlled trial. Clin Nutr., 2018;37(4):1202-1209.

23. VOLLBRACHT C, et al. Blood concentrations of vitamins B1, B6, B12, C and D and Folate in palliative care patients: Results of a cross-sectional study.J Int Med Res., 2019;47(12):6192-6205.

24. WORLD HEALTH ORGANIZATION (WHO). Definition of palliative care. Genebra: OMS, 2002. Disponível em: https://www.who.int/news-room/fact-sheets/detail/palliative-care. Acessado em:10 mar. 2021. 